vision of specific remedial physiotherapy for a short period a day in the early stages with the progressive addition during convalescence of physical training, occupational therapy and mental exercise so that gradually the whole day is devoted to the process of recovering the physical and mental capacity for a full day's work. For example a patient in the later stages of convalescence from a severe pneumonia might spend the final days in hospital in the following manner. 9-Io ward duties, I0-I0.30 standing chest class, I0.30-II break, II-I2 lecture, I2-I lunch, I-3 workshops or gardening, 3-4 standing chest class followed by games, 4 tea. In this manner a few days extra are spent in hospital in getting thoroughly fit instead of leaving at the earliest possible moment and spending an indeterminate convalescence at home. It is not necessary to live in hospital for the final stages and, indeed, the greater contentment of being able to live at home is a positive contribution to rehabilitation; but facilities must be available for the patient to spend the whole day at the rehabilitation centre and to have a midday meal and rest.

\title{
SURGERY IN OLD AGE
}

\section{By D. LANG STEVENSON, F.R.C.S. (Assistant Surgeon, Essex County Hospital, Romford)}

The ability of an old person to stand an operation is not infrequently underestimated because undue significance is given to numerical age, and the tendency to let this play an over important part in the assessment of stamina denies some of the more tough a reasonable chance of cure or relief from some distressing complaint.

Just as old age bears no constant relation to decline in physical fitness or mental acuity, in the same way it is no reliable measure of operability. In the evening of human lifetime strong contrasts become apparent, distinguishing those who have treated their bodies well and who possess the inherited longevity of sound stock from those who have abused health, or who have been unfortunate in their inheritance. An example of this is often seen in the " prostate ward," where an alert fresh-complexioned octogenarian has a companion in local pathology a veritable human derelict who may be fifteen years the junior. It is therefore important both to recognise that those old patients bearing testimonials of long healthy lives, similarly enjoyed by their parents, have remarkable powers of tolerating surgical trauma, and also that those of poorer fibre can be more often escorted safely through surgical illness if the management of their case is modified to suit the needs of frail old age.

Frank evidence of inadequate powers of healing or resistance to infection is not frequently seen in old patients, but there is little doubt that tissue vitality is reduced by a variable degree of cellular atrophy and diminution in the quantity and quality of the blood supply. The same measures which may be effective in making good some of these deficiencies in the vitality of ageing tissues have a considerable value in preventing the post-operative complications, pulmonary infection or oedema, thromboses, and renal failure, peculiar to those advanced in years.

The widespread ill-effects which Penton, Stephenson and Korovensky (I94I) have shown may be caused by deficiency of vitamin $B$ and $C$ in old persons, and the important part that vitamin C (Hunt,.I940) plays in wound healing cân leave no doubt as to the advisability of giving adequate amounts of these vitamins before and after operation. In the opinion of these workers senile vitamin deficiency is not only caused by the low vitamin diet common amongst old people, but is also influenced by defective absorption. They therefore recommend large therapeutic dosage. Tissue anoxia favoured by emphysematous lungs within a fixed thorax and a sluggish circulation within a rigid arterial system can be remedied to a worth-while extent by maintaining bodily movements in the form of regular limb and breathing exercises, and by inhalations of oxygen through a B.L.B. mask during the early post-operative period. Helping these old patients out of bed into a chair on the day following operation insists on a valuable increase in respiration and circulation rate. Stasis can be further prevented by careful attention to water balance, and in certain cases by the controlled administration of thyroid extract. The latter has the noticeable effect of livening up some of these old patients as if the stimulation to metabolism was shared by the waning endocrine system.

The cost of heparin prohibits its general use, but in selected cases prophylactic administration during the first post-operative week is an additional safeguard. 
The value of one or more transfusions for old persons about to undergo major surgery, especially for carcinoma, requires particular emphasis. Sulphanilamide therapy seems to be as effective in the treatment of surgical infections and lung complications in old as in younger patients, but probably shorter courses are advisable on account of the usually low leucocyte response. Ultra-violet ray light therapy is of additional value in promoting a feeling of wellbeing. In my experience attention to these points, which are embodied in the following briefly outlined regime, has made the old subject safer for surgery.

\section{BEFORE OPERATION}

In general, hospital menage should as far as possible be in harmony with the usual habits or fancies of patients. They do not take kindly to certain stringencies in customary routine, such as exhausting investigations, nothing-by-mouth for periods after gastro-intestinal operations, recumbency after herniorraphy, etc., and these should be avoided. If the operation is an elective one, a week or preferably more should be spent in bringing general condition to optimum. The diet contains if possible raw egg beaten up in milk, broth and wine or stout; stout having been found of particular value. In addition, the following are given daily: 500 mgs. vitamin $C$; 5 mgs. aneurin; 50-100 mgs. nicotinic acid; 12,000 units vitamin A. In certain cases thyroid extract is given in suitable dosage. The patient gets up every day if able, and limb exercises are carried out under the supervision of the massage department, together with U.V.R. therapy if available. A high fluid intake is especially encouraged, a daily check being kept. Mild aperients are given, but no routine pre-operative enema. A hot drink is allowed two hours before operation.

\section{CHOICE OF ANAESTHETIC}

Morphine gr. $\frac{1}{4}$ has been found to be the most satisfactory routine premedication, and this is given half an hour before operation. Mental confusion produced by, hyoscine compounds makes their use inadvisable. Local anaesthesia, in combination with intravenous pentothal, and in selected cases, regional subarachnoid block have been found to cover the entire field of surgery in old age with a high degree of safety. The barbiturate drug is injected in quantities of 2 c.c. at a time into the flow of a continuous intravenous infusion of 5 per cent glucose, which, besides having other advantages, greatly facilitates the rapid administration of a stimulant should the need be urgent. An interval of some minutes follows each injection of 2 c.c. until narcosis is induced, when regional anaesthesia of the operation field is carried out, using $\frac{1}{2}$ per cent procaine solution. This method of anaesthetising old patients can be strongly recommended. In selected " over seventies," particularly in cases where relaxation is required, as in colon resections, regional subarachnoid block (Lake, I938) employing light percaine is invaluable. The method has given increasing evidence of its safeness and effectiveness, the amount of this unusually non-toxic drug required for abdominal operations in old people being very small indeed -5 c.c. of $\mathrm{I} / \mathrm{I} 500$ percaine is the average dose, equivalent to $0.003 \mathrm{grm}$. of the drug.

\section{OPERATION}

It goes without saying that the shorter the time an old patient is on the operating table the better. Great care is necessary to see that prominent parts are not damaged by pressure on trolleys or theatre tables, as such injury may be the starting-point of sloughing skin which later retards convalescence. If any principles in surgery of old age are of first importance they are a degree of conservatism compatible with the purpose of the operation, speed, gentleness, and simplicity of procedure. The possibility of slow healing calls for particular attention in the sewing up of wounds, interrupted silk sutures being most satisfactory in clean cases. Transverse incisions are preferable for the "early-up " regime, as they cause remarkably little postoperative pain and have little tendency to disrupt; they seem to heal better than vertical incisions and are easier to sew up under light anaesthesia, especially when aided by raising the upper trunk and lower limbs. 


\section{AFTER OPERATION}

It is of great importance that the patient should be properly covered up whilst in transit from the theatre back to bed, as it is during this journey through cold corridors that an upper respiratory chill may be contracted which is only too swiftly followed by bronchopneumonia. On return to the ward the semi-Fowler position is adopted, and inhalations of oxygen are started through a B.L.B. mask every two hours for fifteen minutes during the first twelve waking hours, and thereafter twice a day for the next few days. Tea, for which old persons crave, is given as soon as it is wanted, and fluid diet is commenced soon after in the form of hot milk, with the addition of brandy if possible, or ovaltine, etc. Nembutal or allonal are amongst the most satisfactory soporifics for senility. Opiates are rarely necessary as the diminished tone of atrophic muscles minimises post-operative pain in old age, and hyoscine compounds should not be given at all. On the day following operation the patient is got out of bed into a chair for a short period unless there are definite contra-indications, e.g. peritonitis with drainage. Each day the time up increases, and limb exercises are carried out under supervision. The resulting movement is not only highly beneficial to the respiratory and circulatory systems and to the tissues, but is also an effective ally in preventing the easily developed senile bedsore. Continuous intravenous infusion requires, of course, particularly careful control in the old subject, but is nevertheless invaluable in certain cases for supplementing inadequate drinking after excessive fluid loss, or for maintaining renal secretion and supplying nourishment. No evidence of undue strain on the senile cardiovascular system has been observed when the infusion is given slowly (30 drops per minute) at body temperature, when careful intake and output charts are kept, and when the infusion is discontinued as soon as a response has been achieved. No splinting of limbs is necessary or desirable during intravenous therapy, and thromboses become a rare complication if strict asepsis is observed, and if no stronger solution than 5 per cent glucose in saline is used for no longer period than twelve hours. Difficulty of micturition is often overcome by getting old men to stand beside the edge of the bed during the act. However, as it is by no means uncommon for prostatic enlargement to precipitate bladder obstruction after operations, especially for associated herniae, and as renal failure has occasionally drawn attention to unsuspected overflow incontinence, the cause of the trouble must be recognised early, and bladder drainage instituted until further treatment can be safely carried out. Suspected or anticipated pulmonary infection has been treated with good effect by $\frac{1}{2} \mathrm{oz}$. of brandy and I gm. of sulphapyridine 4 hourly, and digitalis prescribed by the physician. This treatment has appeared to pull a number of old people through a dangerous period in their illness.

\section{ELECTIVE PROCEDURES}

Although it is urged that surgical cure may be more often attempted in old age with a reasonable chance of success, it is clear that if elective surgery is being contemplated the risk must be carefully balanced against the probable benefits, the degree of interference in the enjoyment of life before operation against the comfort or relief it affords subsequently, and the expectancy of life without surgery against that which might be expected after the operation had been successfully accomplished. As shock and loss of blood are badly tolerated by the aged, it is essential that the extent of certain standard surgical procedures be modified, but fortunately senile pathology tends to favour conservative surgery. The mortality rates of different operations on patients of seventy years and over are difficult to compute, but there are a sufficient number of recorded recoveries (Thewlis, I94I) from major operations to indicated that old subjects are often better operative risks than they are generally believed to be. Some brief notes are given below illustrating some points relevant to the scope and safety of surgical treatment in the commoner operable conditions of old age.

Carcinoma of the stomach in the seventh decade is often a small sclerosing growth at the pylorus which is frequently heralded by obstructive symptoms. Gastro-jejunostomy gives considerable and surprisingly prolonged relief in these cases with little operative risk. Six consecutive patients of over seventy have had the benefit of this operation, which has been performed under local anaesthesia without mortality. One of these patients, an old man of seventy-five, who was up in a chair two days after operation, drew attention to his bilateral inguinal herniae and insisted that these should be surgically cured before he left hospital! In three cases where the stomach was markedly ptosed, the pylorus thus lending itself to simple 
resection, a pylorectomy was performed. Two of these patients recovered and were well three months later. Other much more striking recoveries from partial gastrectomy have been recorded in patients of over eighty (Rowntree I93I, Maingot I942, Gillespie I942).

Although carcinoma of the right colon in senility tends to give the clinical impression of inoperability on account of the marked secondary or toxic anaemia, the ominous lump, and advanced years, laparotomy not infrequently reveals a resectable growth. By means of blood transfusions which improve the general condition considerably, and the adaptation of the PaulMikulicz principle to right hemi-colectomy (Lahey, I932, I939; Devine, I940; Maingot, I942), which greatly reduces the time of operation and the risk of peritonitis, the removal of such a growth in a frail patient has a greater chance of success. Personal experience of this method of resection is limited to only two cases, aged 68 and 72 , who made good recoveries.

The Paul-Mikulicz operation for carcinoma on the left colon carried little risk, and is therefore particularly suitable for old persons. However, taken as a group, " over seventies" suffering from this disease are poor risks as most of them are first seen with subacute or acuteon-chronic obstruction. Finsterer (I936) give a mortality rate of 20.4 per cent in I32. resections, 25 of which were over seventy years of age. From Wilkie's figures (I934) it is seen that a lower mortality rate is obtained by preliminary drainage of the. bowel than that shown after primary partial colectomy by the Paul-Mikulicz or other method. The two or three weeks following colostomy can contribute a great deal to the uneventful recovery of these old patients from the succeeding operation, if every effort is made to improve general condition along the lines previously described. Local infection, oedema and distension of the bowel are greatly reduced by the usual daily tap-water washouts.

Operable growths near the pelvi-rectal junction insufficiently mobile for an exteriorisation procedure appear to be frequently treated in old patients merely by an inguinal colostomy. A transverse colostomy is often preferable for, if the general condition improves sufficiently under treatment, Devine's method of performing abdominal resection on clean bowel followed by end-to-end anastomosis or Hartman's operation can be safely carried out.

Lockhart-Mummery (I933) has shown that perineal resection for carcinoma of the rectum can be more than a palliative operation, and is particularly suitable for old people. In his expert hands a low operative mortality of 8.5 per cent has been obtained in 200 resections, nearly half of which were over 60 years of age and thirty of them over 7o. Recently I saw how the perineal operation in an old patient could be performed rapidly in a more or less devascularised field by preliminary ligature of the superior haemorrhoidal vessels when making the inguinal colostomy. Milligan (I942) has pointed out that more of these frail patients may be submitted to the more radical abdomino-perineal resection with a reasonable chance of survival and a decreasing mortality rate as experience increases in the combined simultaneous operation in which the work is divided between two surgeons. Milligan emphasises that not only is the time of operating and the shock reduced by this procedure, but also more advanced growths previously considered inoperable may be tackled successfully. The safety of this radical operation may be further increased by adopting Lahey's two stage technique (r939) whereby the great advantages of a preliminary working colostomy and a defunctioned rectum are obtained. Hot rectal washouts at $I I 5^{\circ} \mathrm{F}$. given twice daily per rectum, are most effective in reducing the inflammatory induration round the ulcer and the rectum, and an increase in mobility of the growth is not infrequently detectable on rectal examination after this treatment. As septic absorption from the large raw area is a common cause of death in these old subjects, a clean defunctioned bowel and meticulous care in closing the stump with the additional safeguard of sulphaniłamide insufflation into the wound, materially diminish this risk.

It is clear that the indications for cholecystectomy at this time of life must be more urgent than the mere presence of gallstones or a flatulent dyspepsia. However, when severe recurrent colic, inflammation or jaundice has occurred, the relief afforded justifies the operation which at the same time removes the likely cause of more serious complications. Quigley (I939), reporting on cholecystectomy in the elderly, shows a mortality rate of 10.5 per cent in I9 male cases and $I \cdot 9$ per cent in 52 female cases. He also shows that the mortality rate is quite appreciably increased in those cases where common duct exploration is necessary. Thorek's modification of cholecystectomy by electro-coagulation is of much value in the often shrivelled and partially intrahepatic diseased gall bladder of old age. This method was found of considerable value in eight cases out of fifteen cholecystectomies which I performed on a series of old patients of both sexes whose average age was sixty-seven (one post-operative death). As it is of much 
importance that post-operative pain should not interfere with easy breathing a transverse incision, which seems to produce minimal discomfort, is preferable.

No patient need be refused repair of their herniae solely on account of age. Watson (I938) has particularly emphasised this view. Contra-indications are as a rule to be found in the buge size of the hernia or the attenuated condition of the abdominal wall. Herniae are often initiated or aggravated by chronic bronchitis or, in old men, by straining at micturition due to enlargement of the prostate which should first receive attention. In respect of the former it is well to remember the benefit afforded to these old patients if the operation is undertaken in the warm season. Many very old people have recovered from herniorraphy, and several outstanding examples of recoveries in the ninth decade have been recorded. Coulson (I942) reported the case of a man who died recently at the age of ninety-eight, upon whom he had operated for a strangulated umbilical hernia seven years previously. As femoral and umbilical herniae have a high potential danger of strangulation, operation should be advised in the aged unless the size of the latter hernia precludes the possibility. Even very large-sized inguinal herniae may be repaired satisfactorily by the muscle slide operation described by Tanner (I942).

Numerous successful operations for goitre in the aged have been recorded. Of considerable interest is the recognition of milder degrees of toxic goitre in old persons who have greatly improved after subtotal thyroidectomy (Keynes, I937). Cattell (I94I) calls attention to some important points relevant to this elderly group of thyroid patients: (I) that many of them are treated as "heart cases" because of persistent tachycardia or evidence of decompensation; (2) that the diagnois is made on the findings of a firm thyroid gland, a fine tremor, together with weight loss in spite of a, reasonable food intake, and a moderate but consistent increase in the basal metabolic rate on repeated tests; (3) that in most cases it is advisable to accomplish subtotal thyroidectomy as a staged procedure, allowing six weeks to elapse between dealing with each lobe, and four weeks to elapse after a preliminary pole ligation if that has been considered an additional necessary precaution; (4) that the administration of Lugol's solution, which is commenced between two and three weeks before operation, should be continued throughout the entire period.

Senile carcinoma of the breast illustrates well how the malignancy of the disease may be greatly reduced when it invades aged tissues. The treatment of choice is often, therefore, either local excision of the breast by diathermy or the insertion of interstitial radium.

Age alone need not stand in the way of a comfort-restoring perineal repair for a distressing procidentia. My gynaecological colleague, Mr. Henderson, supports this view, and has had several most satisfactory results in women of over eighty. He also quotes a further interesting case of an old lady of eighty-four who was bedridden for two years because she was believed to suffer from ascites due to heart failure. The removal of a large ovarian cyst under local anaesthetic cured her " heart " trouble and granted her great desire of being able to get up and about once more.

Experience of old men suffering from enlargement of the prostate has shown that selection is of great importance, for they can be divided roughly into two groups: (I) those in whom the bladder obstruction is the only factor interfering with otherwise reasonable good health, and (2) those who are human derelicts with ill-masked cardio-vascular and renal impairment. The latter group, which are seldom suitable for suprapubic prostatectomy, even after prolonged bladder drainage, raise the death-rate at any clinic where they are frequent candidates for this operation. It is to this group of old men that the perurethral operation by means of the punch resectoscope may hold out new hope. Wardill (I94I) has pointed out that Gershom Thompson and his colleagues in the Mayo Clinic practise the operation with a very low mortality rate (45I cases without a death), and with good functional results. Wardill has reported a mortality rate of 10.4 per cent in a personal series of 230 cases, many of which were poor risks. He also draws attention to the fact that these patients are out of bed on the first or second day, and that the average time of hospitalisation is twelve days. Chapman (I943) in a recent article also gives most interesting figures (six deaths in a hundred cases), and emphasises the value of the perurethral resection in the poor risk. By increased experience with this instrument (which seems to require considerable and careful practice), and by using it more extensively, function may be restored quickly and safely to these old men with little need for selection, and many hospital beds would at the same time be released.

The introduction of the Smith-Petersen pin has made the common fracture of the neck 
of the senile femur, with its spectre of fretting and fatal recumbency, less of a major disaster for these old people. Selection is of course necessary to exclude unsuitable subcapital fractures and extreme examples of senility, but if the condition is reasonable, a painless mobile hip is a sufficient blessing to an old person to justify the time and trouble of a skilled surgeon, whatever the age or statistical expectancy of life. Watson Jones (I94I) gives such an opinion, and cites two patients, aged ninety-one and ninety-two, on whom he operated, and who were leading happy lives one and five years after operation. King (I939) reports sixteen such operations on patients between eighty and ninety-eight years of age with only two deaths. My former chief, Mr. A. E. Freeman, of Wolverhampton, has had a most successful result following the introduction of a Smith-Peterson pin in a woman of ninety-eight.

Fractured ribs may prove a serious injury in old patients, as the acute pain may so inhibit normal respiration, and favour the accumulation of infective secretion that, together with. some slight visceral trauma, broncho-pneumonia is precipitated. Strapping, although it relieves the pain to some extent, also interferes with the respiratory excursion. The most satisfactory treatment is that recommended by Neil Smith (I942), who injects Io c.c. of I/IOoo percaine into and around the sites of the fractures. I have used I per cent procaine in combination with proctocaine for several years in these cases, and I have also found it effective. The pain is greatly relieved, and an old patient can usually walk in comfort with some assistance shortly after the injections.

When all efforts to save the limb with senile gangrene have failed, amputation may be performed without pain or any evidence of shock, and with the minimal chance of infection by freezing the limb for three or four hours before operation and for some hours after. A low mortality rate of I death in 30 poor risk cases has been reported by Allen et al (I942). The value of the method is indicated in the case of an old man of eighty-five both of whose lower extremities were literally dead from below the knee and intensely painful. Although his general condition was precarious there was no alteration in his pulse or general condition during bilateral mid-thigh amputation or afterwards. He was most grateful for the relief from the agonising pain which he had endured for a long time, and as a result he enjoyed the Christmas ward festivities as heartily as anyone.

It is interesting to learn from the ophthalmic department that the results of cataract operations in the aged have noticeably improved since a few weeks have been spent in bringing general condition to an optimum by particularly stressing a high vitamin diet. It is also interesting to learn from a colleague of a successful cataract removal in a patient of ninety-nine.

\section{EMERGENCY PROCEDURES}

The mortality rate of abdominal emergencies in old subjects is higher than it need be, as too often they are sent into hospital too late. Douglas (1942) and others have shown that the increasing time factor is directly related to the rise in the mortality rate of strangulations. Unlike the complicated external hernia, the acute intra-abdominal emergency in the aged often provides a difficult diagnostic problem. Little or no rigidity due to atrophic muscles, the absence of vomiting, and a normal temperature, which are characteristic of the acute senile abdomen, can effectively mask the gravity of the underlying lesion. It is also a well-established clinical observation that in the early hours after perforation of a peptic ulcer the pulse is not uncommonly slow. For these reasons it is safer to send these old patients to hospital early for observation when the diagnosis is doubtful. Referred pain from heart disease is a common stumbling block in the differential diagnosis, but it is frequently noticeable that these elderly cardiacs have, amongst other distinguishing clinical features, faces and extremities which are cold to touch and also a subnormal temperature.

Strangulated femoral herniae in aged persons are dealt with most expeditiously by division of Pouparts ligament and the method of repair recommended by Hey Groves (I922), which is particularly valuable if resection is necessary. Doubtful viability of bowel at the constriction rings can be safely treated by oversewing with interrupted Halsted sutures of fine silk. In my experience, if the loop of gut is clearly beyond hope, then resection and primary anastomosis gives these old patients a better chance of recovery than any exteriorisation procedure, whatever their condition. Among several examples of very old patients who have made good recoveries after resection of gut there is the striking case of an old lady of eighty-one who was discharged from hospital ten days after removal of 12 inches of bowel and the repair of a femoral hernia: It is particularly important that these old people are got out of bed early after operation. 
It is common experience that aged persons make good recoveries from operations for acute appendicitis or perforated ulcers if they are brought under surgical care soon enough after the onset of the catastrophe. In my own cases the oldest recovery from a perforation was eightyseven, and from appendicitis eighty-four. On account of the mild clinical picture that may be associated with fulminating acute appendicitis, the delayed, or Oschner, treatment is seldom justified.

It has been my experience that acute obstructive cholecystitis is not as amenable to conservative treatment in the elderly as it is usually found to be in the younger patient, and that early cholecystostomy is, therefore, generally advisable. There is one type of small bowel obstruction peculiar to old age which is worthy of mention, namely, gallstone obturation. The symptoms are usually colicky central abdominal pain and much vomiting, with well-marked intermittent periods of relief, varying in duration. Distension or absolute constipation are rarely present, although visible peristalsis is occasionally seen. This clinical syndrome, coupled with the fact that the medical attendant has usually been long aware of the troublesome gall bladder, makes the mistaken diagnosis of biliary colic a frequent happening, and probably explains the high mortality rate (40-50 per cent) in a condition easily remedied by surgery. The value of the indwelling duodenal tube and the replacement of fluid and chlorides in all serious cases of intestinal obstruction needs no enlargement.

The inadequate time allowed for the preparation of an old patient with an abdominal emergency calls for increased care during and,after operation, speed in accomplishing what is surgically necessary, the use of local anaesthetic, continuous intravenous infusion (to which in the case of a poor risk it is an advantage to add 5 c.c. of coramine or $I-I \frac{1}{2}$ c.c. adrenalin $I / I 000$ sol. to I litre of saline), and local and general sulphanilamide therapy, are important factors in increasing the safety of the operation. Alcohol is of considerable value in all serious cases.

\section{SUMMARY}

Many old patients are better subjects for operation than they are generally believed to be. If the surgical management is modified to meet their special requirements, the hazard of an operation is appreciably reduced, and fewer need be denied the extended life of increased comfort which surgery may offer.

Some of the commoner operable conditions in old age have been considered very briefly in terms of scope and safety

As it has been shown that I5 per cent of the population in forty years' time will probably be over sixty-five years of age, it is clear that the study and treatment of disease at this time of life will become increasingly important.

My grateful thanks are due to Mr. Rodney Maingot, F.R.C.S., for a great deal of helpful advice and criticism, and to Dr. Miles, Medical Superintendant of Oldchurch Hospital, for permission to cite some of these cases.

\section{BIBLIOGRAPHY}

STEPHENSON, W., PENTON, C., KOROVENSKY, V. (1941), B.M.J., I941, 2, 893.

HUNT, A. H. (I9OO), B.J.S., $28,436$.

LAKE, N. C. (1938), Lancet, I938, 1, $24 \mathrm{I}$.

THEWLIS, M. W. (194I), Care of the aged, Henry Kimpton, xxxviii, 553.

ROWNTREE, C. (I93I), Clin. Journ., 60, 257.

MAINGOT, R. (I94I), Abdominal Operations, Appleton Century, London, I. 363 (1942), Medical Press and Circular, September 9th.

GILLESPIE, M. G. (1942), Amer. Journ. Surgery, 57, 318 .

LAHEY, F. (1932), Surg. Gyn. Obstet., 54, 923, and (1939) Amer. Journ. Surg., 46, 3.

DEVINE, $\dot{H}$. (1940), Surgery of the alimentary tract, Wrights, Bristol, $8 \mathrm{i}, 900$.

FINSTERER, H. (1036), Amer. Journ. Cancer, 28, 198.

WILKIE, D. P. D. (1934), Lancet, 1, 65.

HARTMANN, HENRI (1923), Congres Franjais Chir., 30, $4 \mathrm{II}$.

LOCKHART-MUMMERY, J.' (I933), Amer. Journ. Cancer, 18, I.

MILLIGAN, E. T. C. (1942), Procs. Roy. Soc. Med., 35, 77 I.

QUIGLEY, T. B. (I939), New Eng. Journ. Med., 221, 970.

WATSON,' L. F. (1938), Hernia, Henry Kimpton, 2, 107.

TANNER, N. C. (I942), Brit. Journ. Surg., 30, 285.

KEYNES, G. (I937), Practitioner, July, 44, 139 .

CATTELL, R. B. (1941), Pennsylvania Med. Journ., 44, 685.

WARDILL, W. E M. (I94I), Lancet, 2 , 127.

CHAPMAN, T. L.' (1943), Lancet, I943, 1 , I4.

WATSON JONES, R. (I94I), Fractures and other bone and joint injuries, E. \& S. Livingstone, Edinburgh, 460.

KING, T. (1939), B.J.S., 26, 722 .

ALLEN, F. M., CROSSMAN, L. W., HURLEY, V., WARDEN, C. E., RUGGIERO, W. (1942), International Coll. of Surg. Journ., DOUG'I25.

D. M. (1942), B.M.J., 1, 354

WILCOX, L. E., CLAGETT, O. T. (I94I), Proc. of the Mayo Clinic, 16, 795. 